\begin{tabular}{|c|c|}
\hline Title & Magnetic circuit modeling of chaotic vibration energy harvester \\
\hline Author(s) & Maruo, A kito; Igarashi, Hajime \\
\hline Citation & $\begin{array}{l}\text { International journal of applied electromagnetics and mechanics, 59(2), 567-575 } \\
\text { https://doi.org/10.3233/AA E-171248 }\end{array}$ \\
\hline Issue Date & 2019-03-21 \\
\hline Doc URL & http:/hdl.handle.net/2115//3860 \\
\hline Rights & The final publication is avail able at IOS Press through http://dx.doi.org/10.3233/JA E-171248 \\
\hline Type & article (author version) \\
\hline File Information & ISEM2017_full_maruo_ver7_submitver.pdf \\
\hline
\end{tabular}

Instructions for use 


\title{
Magnetic Circuit Modeling of Chaotic Vibration Energy Harvester
}

\author{
First Author and Second Author \\ Address of the First and Second authors usually few lines long
}

\begin{abstract}
The chaotic vibration energy harvester (CVEH) converts vibration of constructions to electric energy in wide frequency. It needs large computational cost for evaluation of the magnetic force between the moving magnets and magnetic material embedded in the stator coils of CVEH when using finite element method (FEM). This paper presents magnetic circuit modeling of CVEH to reduce computational burden. It is shown that the frequency responses of CVEH computed by the present method is in good agreement with those obtained by FEM. This result is validated through the experiment.
\end{abstract}

Keywords: Vibration Energy Harvester, Magnetic circuit, Chaotic oscillation, Genetic algorithm

\section{Introduction}

The vibration energy harvester (VEH) which converts vibration of constructions into electric energy. It has attained great attentions for the energy sources of low-powered wireless sensor devices composed of sensors, interface and communication ICs [1,2]. A conventional VEH works only at around the resonance frequencies, whereas the actual vibration can have wide frequency spectra. To overcome this difficulty, we have proposed the chaotic VEH (CVEH) which works in wider frequency ranges [3, 4]. In CVEH, the potential energy whose derivative corresponds to the magnetic force between the permanent magnets and the silicon steel plates embedded in the coil has a double-well structure. The nonlinearity in the magnetic force with respect to the displacement leads to the operation in a wide frequency range. Moreover, when the design parameters are carefully tuned, it is found in numerical results and experiments that chaotic vibrations emerge.

In design of $\mathrm{CVEH}$, the magnetic force has been evaluated using three-dimensional finite element method (FEM) [5]. However, FE analysis which include mesh generation for considering movement of the oscillator needs large computational cost. In this paper, we propose magnetic circuit modeling of CVEH to reduce computational burden. The circuit parameters, some of which are the function of displacement, are identified so that the magnetic potential is as close to that computed by FEM as possible. Once the circuit parameters are identified, we can computed the magnetic potential for different design parameters. We can numerically analyze the magnetic potential of CVEH using the magnetic circuit without time-consuming FE analysis. It will be shown that the chaotic motion of CVEH can be reproduced when using the present magnetic circuit.

\section{Magnetic circuit modeling of CVEH}

Figure 1 shows $\mathrm{CVEH}$ which consists of NdFeB magnets and coils [4]. The magnets are fixed to a cantilever which is oscillated by ambient vibrations. As a result, the magnetic flux across the coils temporally changes, and electromotive force is induced in the coils. The pieces of silicon steel are inserted into the bobbin hole of the coil so as to increase magnetic flux interlinked with the coils. The nonlinear magnetic force with respect to the displacement between the magnets and silicon steel in the bobbins gives rise to nonlinear oscillation of the VEH. This nonlinear oscillation makes it possible for CVEH to realize off-resonant operations. The double coils are introduced so that the magnetic flux forms a closed loop at the two extremes of the oscillation. 


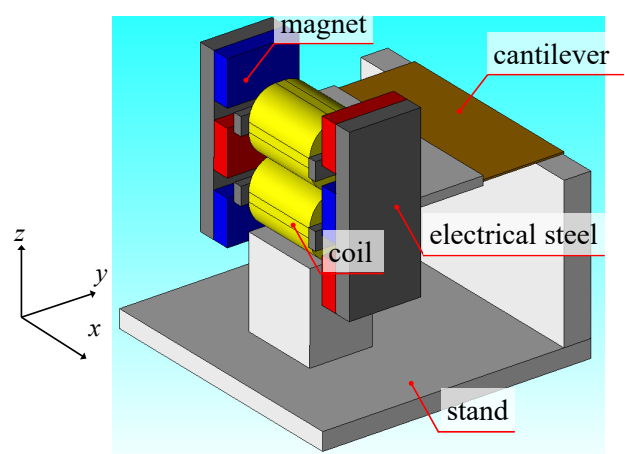

Fig. 1. CVEH model

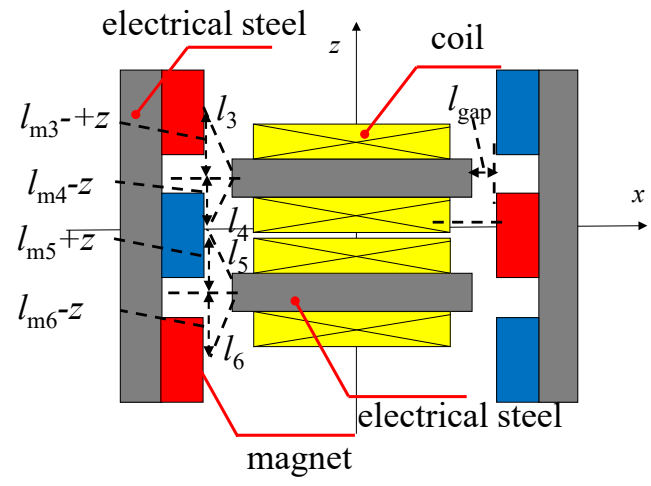

Fig. 2. Cross sectional view of CVEH

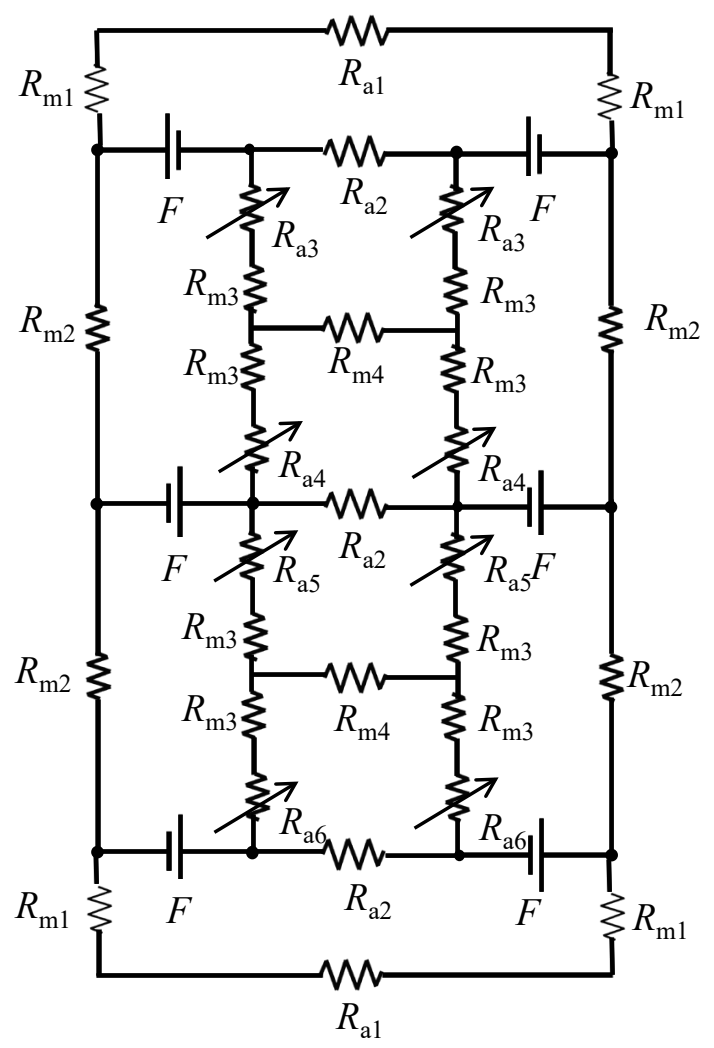

Fig. 3. Magnetic circuit model of CVEH

\subsection{Equation of motion of the $C V E H$}

The equation of motion for CVEH is expressed as

$$
\begin{aligned}
& m \ddot{z}(t)+\gamma \dot{z}(t)+\frac{d E(z)}{d z}=-m \ddot{y}(t) \\
& E(z)=E_{\text {mag }}(z)+\frac{1}{2} k z(t)^{2}
\end{aligned}
$$

where $m, z, \gamma, E$ and $k$ are the oscillator mass, relative displacement between the coil axis and magnets, damping coefficient, potential energy and spring constant, respectively [6]. Moreover, $E_{\text {mag }}$ represents the magnetic energy which is computed from either magnetic circuit model or FEM.

\subsection{Equivalent Magnetic Circuit of $\mathrm{CVEH}$}

Figure 3 shows the magnetic circuit model of CVEH shown in Fig. 2 in which $F, R_{m}$ and $R_{a}$ represent the magnetic force, magnetic resistances of the silicon steel and air, respectively. The magnetic resistances of the air gap $R_{a i}, 3 \leq i \leq 6$, depend on the displacement of the oscillator position $z$. They would be expressed as

$$
\begin{aligned}
& R=\frac{l}{\mu S} \\
& l_{i}= \begin{cases}\sqrt{l_{\text {gap }}^{2}+\left(l_{\mathrm{m} i}-z\right)^{2}}, & (i=3,5), \\
\sqrt{l_{\text {gap }}^{2}+\left(l_{\mathrm{m} i}+z\right)^{2}}, & (i=4,6) .\end{cases}
\end{aligned}
$$

where $\mu$ and $S$ are the parmeability and the cross-sectional area of magnetic flux path. By determining the magnetic flux $\Phi$ in the magnetic circuit, $E_{\text {mag }}$ is computed from

$$
E_{\mathrm{mag}}(z)=\sum_{i=1}^{4} \frac{1}{2} R_{\mathrm{m} i} \Phi_{\mathrm{m} i}^{2}+\sum_{i=1}^{6} \frac{1}{2} R_{\mathrm{a} i} \Phi_{\mathrm{a} i}^{2}
$$


which is inserted into (1) for dynamic analysis of CVEH. The parameters in magnetic circuit, $F, R_{\mathrm{m} i}$ and $R_{\mathrm{a} i}$ are determined so that $E_{\mathrm{mag}}$ computed from the magnetic circuit is as close as possible to that obtained by FEM. For this purpose, we solve the optimization problem defined by

$$
F=\sum_{i}\left|\frac{E_{i}^{\mathrm{FEM}}-E_{i}^{\mathrm{MC}}}{E_{i}^{\mathrm{MC}}}\right| \rightarrow \min .
$$

using genetic algorithm $(\mathrm{GA})$ where $E_{i}^{\mathrm{FEM}}$ and $E_{i}^{\mathrm{MC}}$ are the potential energy $E$ computed form the FEM and the magnetic circuit at the $i$-th sampling point.

\subsection{Improvement of accuracy in magnetic circuit model}

The magnetic circuit should give accurate results for different air gaps because they are tuned so that CVEH provides the maximum output for the given spectra of ambient vibration. It is found, however, that the magnetic potentials computed from the magnetic circuit with the variable magnetic resistances of the air gap $R_{a i}$ given by (3) and (4) result in significant errors when the gap length is changed. To improve the acccuracy, the expression of $R_{\mathrm{a} i}$ is modified as in the following way.

(i) When determining the circuit parameters, the leakage magnetic flux dependent on mutual displacement is taken into account. To do so, we consider three magnetic paths in the air gap-as shown in Fig. 4 , where $l_{i}^{1}$ are the distance between the center of the magnet and the center of the silicon steel in the coil axis.

(ii) When the air gap between the coil axis and magnets increases, the magnetic flux in the air gap extends, which leads to increase in the cross-sectional area of the magnetic flux. The spatial distribution of the magnetic flux in the air gap is considered. From equation (3), it is clear that the value of the magnetic resistance is inversely proportional to the cross-sectional area $S$. This effect is considered in the evaluation of $R_{a i}$.

From (i) and (ii), $R_{a i}, 3 \leq i \leq 6$, are now expressed by

$$
R_{\mathrm{a} i}=\frac{R_{\mathrm{a} i}^{1} R_{\mathrm{a} i}^{2} R_{\mathrm{a} i}^{3}}{R_{\mathrm{a} i}^{1} R_{\mathrm{a} i}^{2}+R_{\mathrm{a} i}^{2} R_{\mathrm{a} i}^{3}+R_{\mathrm{a} i}^{3} R_{\mathrm{a} i}^{1}} \times\left\{1+c\left(\frac{l_{\mathrm{gap}}-l_{\mathrm{gap}}^{\mathrm{min}}}{l_{\mathrm{gap}}^{\min }}\right)\right\}^{-1}
$$

where $c$ is a constant relevant to the dependence of the magnetic resistance on $l_{\text {gap }}$. The minimum value of the flux path lengths of the air gap $l_{\text {gap }}^{\mathrm{min}}$ is set to $1 \mathrm{~mm}$. In the identification of the circuit parameters, $l_{\text {gap }}$ is set to $1 \mathrm{~mm}$ and $1.6 \mathrm{~mm}$. The optimization problem is modified to

$$
F=\sum_{\substack{i \\ l_{\mathrm{gap}}=1 \mathrm{~mm}}}\left|\frac{E_{i}^{\mathrm{FEM}}-E_{i}^{\mathrm{MC}}}{E_{i}^{\mathrm{MC}}}\right|+\sum_{\substack{i \\ l_{\mathrm{gap}}=1.6 \mathrm{~mm}}}\left|\frac{E_{i}^{\mathrm{FEM}}-E_{i}^{\mathrm{MC}}}{E_{i}^{\mathrm{MC}}}\right| \rightarrow \min .
$$

There are deviations in the resultant circuit parameters obtained by solving (8) when starting from different random seeds in GA. We choose the best solution among them. The convergence history to obtain the best solution is shown in Fig. 6 .

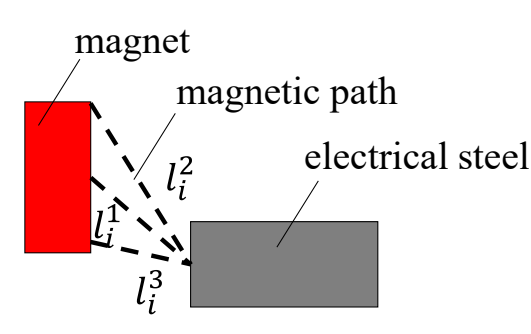

Fig. 4. Magnetic paths in air gap

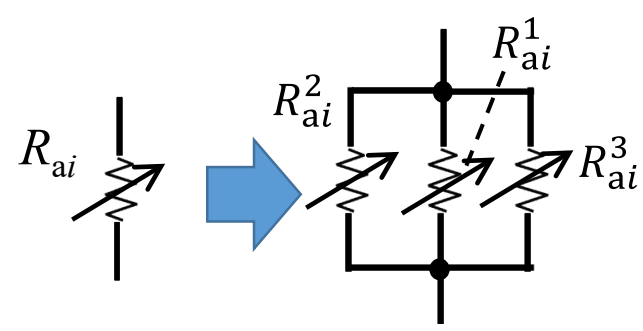

Fig. 5. Magnetic circuit model of air gap 


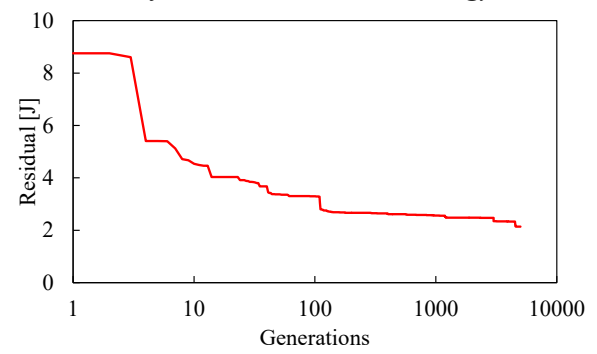

Fig. 6. Convergence history of GA

\section{Analysis of the CVEH}

\subsection{Evaluation of potential energy}

We compute $E_{\text {mag }}$ using the proposed magnetic circuit and FEM. In this analysis, we set damping coefficient $k$ to $1000 \mathrm{~N} / \mathrm{m}$. Figure 7 shows the profiles of the resultant potential energy $E(z)$ for different values of $l_{\text {gap }}$, where conventional MC and proposed MC represent the results obtained based on (4) and (7), respectively. As we can see in Fig. 7, the potential profiles obtained by the proposed method is in good agreement with those obtained by FEM. It is remarkable that the proposed method is valid even when $l_{\text {gap }}$ changes between the two extremes at which the circuit parameters are identified. It would be possible, therefore, to accurately simulate the dynamics of CVEH with small computational cost using the proposed magnetic circuit. To compute these potential profiles, FEM analysis requires a few hours, whereas the magnetic circuit requires a few seconds.

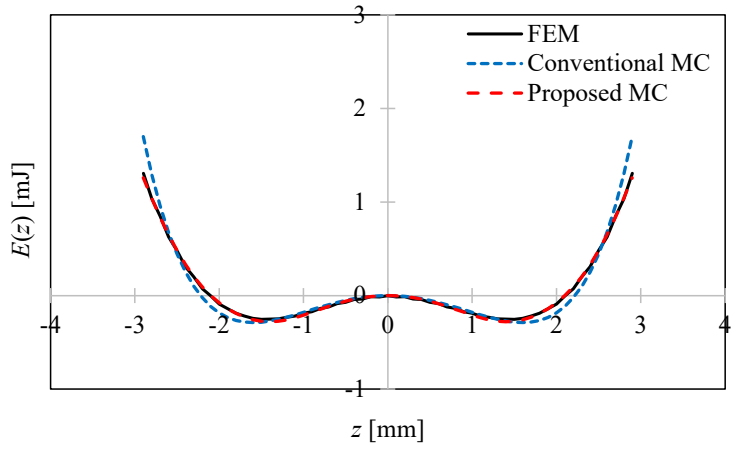

(a) $l_{\text {gap }}=1.0 \mathrm{~mm}$

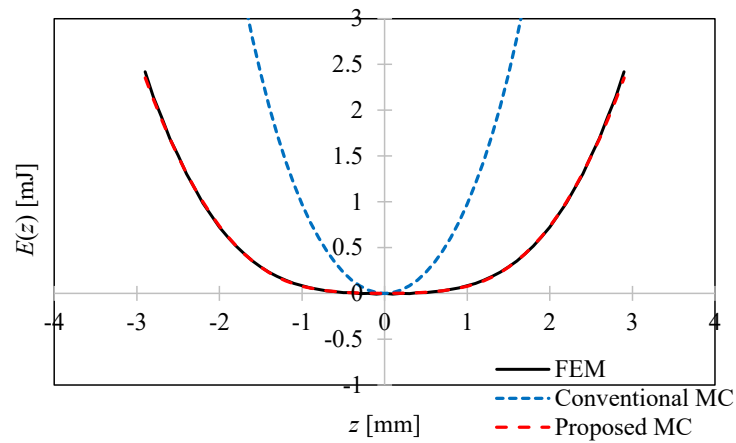

(c) $l_{\text {gap }}=1.4 \mathrm{~mm}$

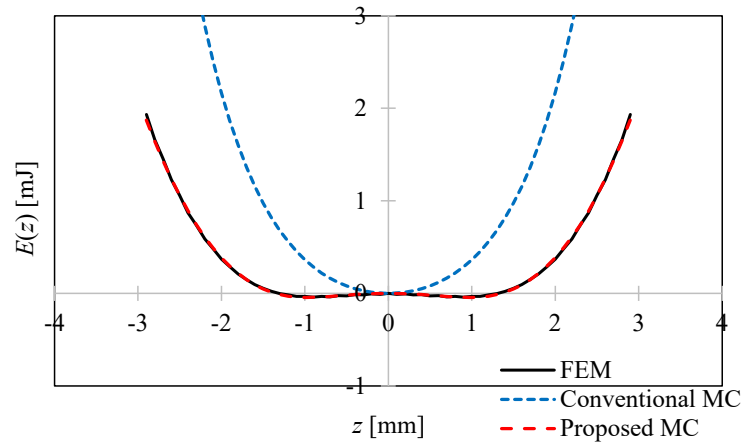

(b) $l_{\text {gap }}=1.2 \mathrm{~mm}$

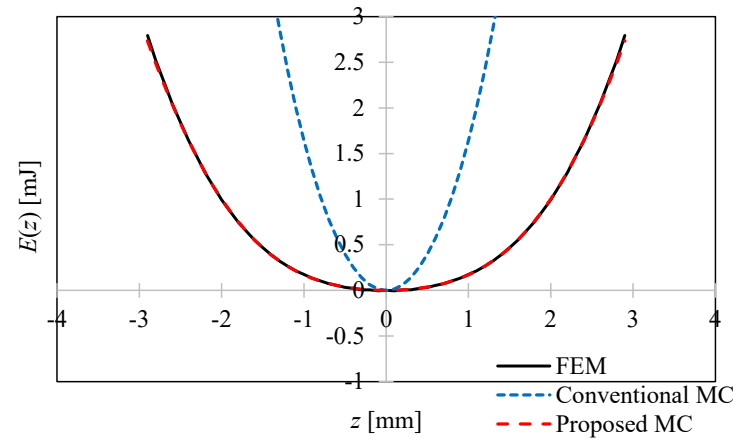

(d) $l_{\text {gap }}=1.6 \mathrm{~mm}$

Fig. 7. Potential energy profiles

\subsection{Frequency response}

We employ the Newton Raphon method to solve (1) to compute the oscillator velocity $\dot{z}(t)$. In this analysis, $m, k, \gamma, l_{\text {gap }}$ and $y(t)$ are set to $17.5 \mathrm{~g}, 0.1,1200 \mathrm{~N} / \mathrm{m}, 1.0 \mathrm{~mm}$ and $A \sin (\omega t)$, respectively. The input acceleration is set to $0.1 \mathrm{G}$ and $1.5 \mathrm{G}$, where $\mathrm{G}$ is gravitational acceleration. The frequency characteristics of CVEH obtained by the proposed method and FEM are shown in Fig. 8. We can find from Fig. 8 that the frequency response obtained by the proposed method is in good agreement with those obtained by FEM. When the input acceleration is $0.1 \mathrm{G}$, the maximum value of velocity suddenly goes up at $18 \mathrm{~Hz}$ and gradually decreases as shown in Fig. 8 (a). This suggests that the oscillator is trapped to one of the wells. When the input acceleration is increased to $1.5 \mathrm{G}$, the velocity has the random variations from $5 \mathrm{~Hz}$ to $13 \mathrm{~Hz}$ and $39 \mathrm{~Hz}$ to $42 \mathrm{~Hz}$, and it increases linearly with 


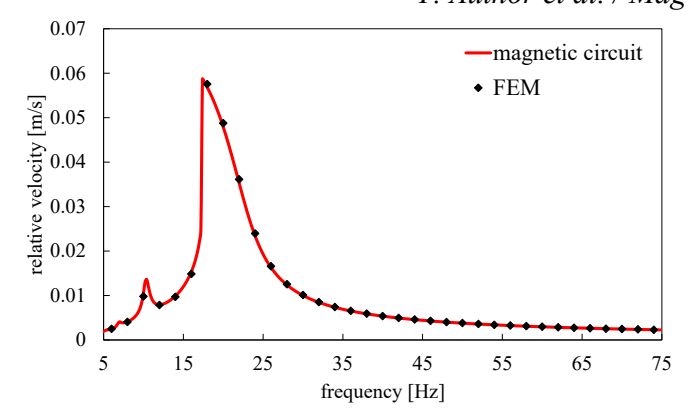

(a) $0.1 \mathrm{G}$

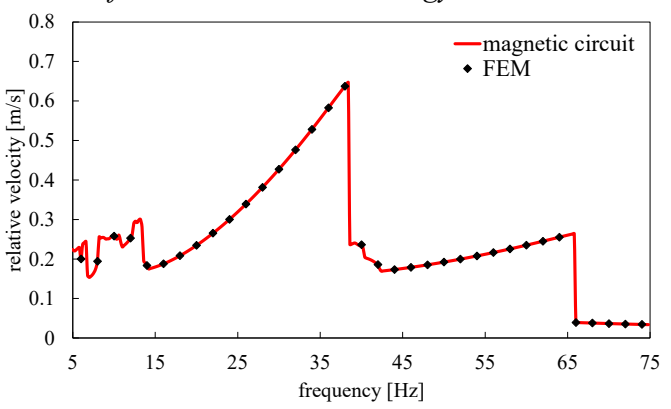

(b) $1.5 \mathrm{G}$

Fig. 8. Frequency characteristics

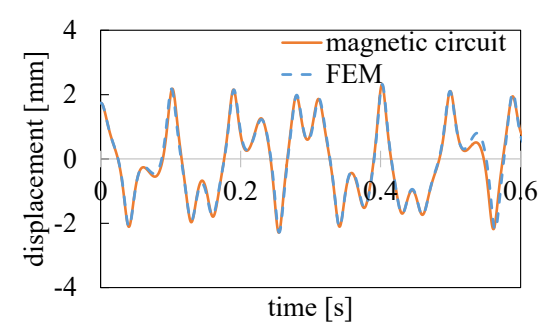

(a) $10 \mathrm{~Hz}$

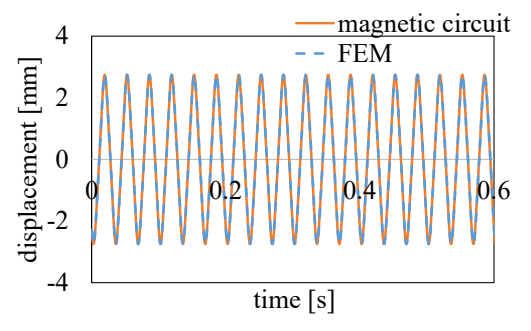

(b) $30 \mathrm{~Hz}$

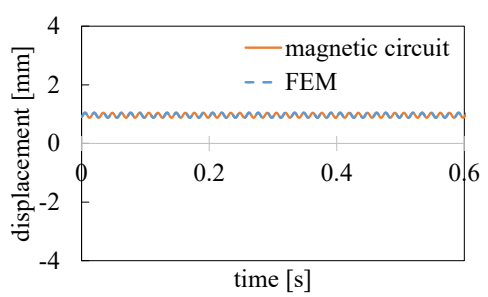

(c) $70 \mathrm{~Hz}$

Fig. 9. Time variations of $z$ computed by proposed magnetic circuit when acceleration is $1.5 \mathrm{G}$

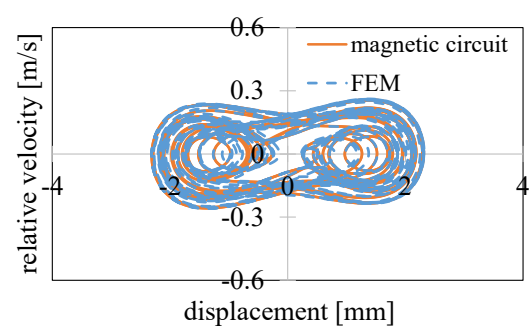

(d) $10 \mathrm{~Hz}$

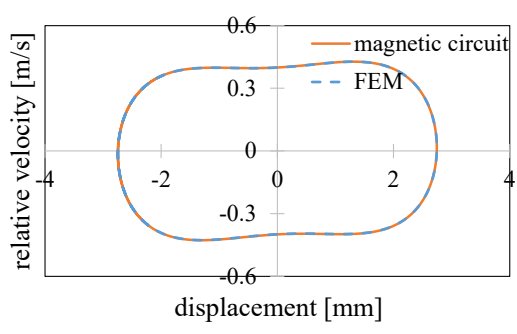

(e) $30 \mathrm{~Hz}$

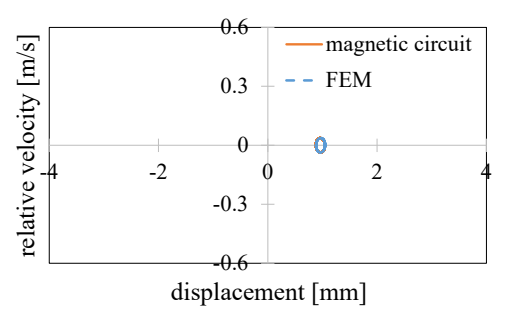

(f) $70 \mathrm{~Hz}$

Fig. 10. Trajectories on phase plane $(z, \dot{z})$ by proposed magnetic circuit when acceleration is $1.5 \mathrm{G}$

frequency from $14 \mathrm{~Hz}$ to $38 \mathrm{~Hz}$ and $43 \mathrm{~Hz}$ to $66 \mathrm{~Hz}$, as shown in Fig. 8 (b). Moreover, it suddenly goes down at $38 \mathrm{~Hz}$ and $66 \mathrm{~Hz}$.

Figure 9 and figure 10 show that the time variations of $z$ and trajectories on phase plane $(z, \dot{z})$, respectively, when the input acceleration is $1.5 \mathrm{G}$. This suggests that the chaotic, interwell and intrawell vibrations are induced at $10 \mathrm{~Hz}, 30 \mathrm{~Hz}$, and $70 \mathrm{~Hz}$, which are also observed in the measurement [3]. That is, the oscillator makes transition between two equilibrium positions of the potential energy when frequency is smaller than $66 \mathrm{~Hz}$, whereas it traps to one of the wells of the potential energy when frequency is higher than $67 \mathrm{~Hz}$. To characterize the vibration at $10 \mathrm{~Hz}$, we evaluate the Lyapunov exponent using Rosenstein method [7]. Its mean value is about 23, from which we conclude that this vibration is chaotic.

\section{Comparison with experiment}

To verify the numerical result, we peform experiment using the manufatured CVEH device shown in Fig. 11 [4], where cantliever and electrical steel are, the phosphor-bronze and JIS 50H400. The frame is produced using a 3D printer (Roland, ARM-10). The number of turns in the coil, which is made of a conducting wire $0.1 \mathrm{~mm}$ in diameter, is set to 1200 . The parameters $m, k$ and $l_{\text {gap }}$ are set to $17 \mathrm{~g}, 500 \mathrm{~N} / \mathrm{m}$ and $1.4 \mathrm{~mm}$, respectively. A resistive load, $100 \mathrm{k} \Omega$, is connected to the coils, and the load voltage is measured by an oscilloscope. The input acceleration is fixed to $0.1 \mathrm{G}$, which is a typical acceleration observed in the constructions [8].

We compare the frequency characteristics of VEH observed in the experiment and computed by the proposed magnetic circuit. Figures 12 and 13 show the computed potential energy and the frequency responses. We can see in Fig. 13 that the computed frequency response has the almost the same 


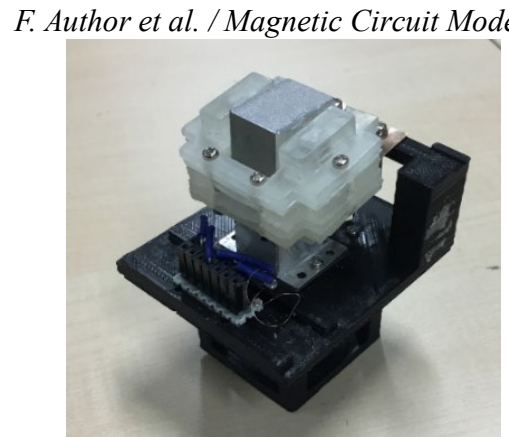

Fig. 11. Manufactured VEH

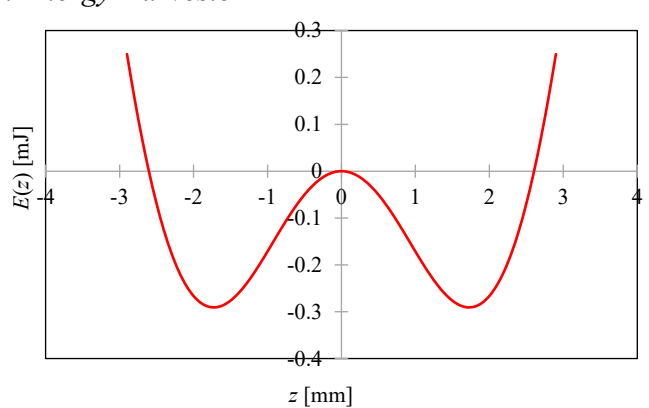

Fig. 12. Potential energy

0.40

0.35

0.30

0.25

0.20

0.15

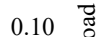

0.05

50

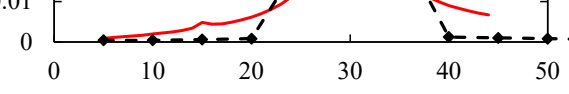

frequency $[\mathrm{Hz}]$

Fig. 13. Measured and computed frequency response

resonant frequency as the measured response has. These vibration mode corresponds to the intrawell oscillation. The computed profile near the resonance frequency is narrower than the measured one. One of the reasons of this difference would be due to the manufacturing error.

\section{Conclusions}

In this paper, we have proposed a magnetic circuit model of CVEH wich can be used for its dynamic analysis. We have improved the accuracy in the magnetic circuit model by considering the change in the air gap length and effective area of the magnetic flux. The proposed method has been shown to be accurate even when the air gap length is changed. The motion of CVEH computed based on the magnetic circuit has chaotic, interwell and intrawell modes being dependent on the input frequency and acceleration. The computed frequency response for $0.1 \mathrm{G}$ has almost the same resonant frequency as the measured one.

The proposed magnetic circuit is effective to tune the response of CVEH to the spectrum of given ambient vibration. Moreover, it is also useful to optimize the design parameters of CVEH to maximize the performance.

\section{Acknouwledgement}

This work was supported in part by KAKENHI $15 \mathrm{H} 02976$.

\section{References}

[1] S. P. Beeby, M. J. Tudor and N. M. White, "Energy harvesting vibration sources for microsystems applications," Meas. Sci. Technol., Vol. 17, pp. 175-195, 2006.

[2] M. Wischke, M. Masur, M. Kröner, P. Woias, "Vibration harvesting in traffic tunnels to power wireless sensor nodes," Smart Materials and Structures, Vol. 20, No. 8, 085014, 2011.

[3] T. Sato, H. Igarashi, "A Chaotic Vibration Energy Harvester Using Magnetic Material," Smart Materials and Structures, Vol. 24, No. 2, 025033, 2015.

[4] T. Sugisawa, H. Igarashi, "Properties of chaotic vibration energy harvester: comparison of numerical results with experiments," Int J Numer Model, doi: 10.1002/jnm.2205, 2016.

[5] T. Sato, K. Watanabe, H. Igarashi, "Coupled Analysis of Electromagnetic Vibration Energy Harvester With Nonlinear Oscillation," IEEE Transactions on Magnetics, Vol. 50, No. 2, 7007604, 2014.

[6] D. A. W. Barton, S. G. Burrow, and R. C. Lindsay, "Energy harvesting from vibrations with a nonlinear oscillator," Journal of Vibration and Acoustics, Vol. 132, 021009-7, 2010.

[7] M. T. Rosenstein, J. J. Collins, C. J. D. Luca, "A practical method for calculating largest Lyapunov exponents from small data sets," Physica D: Nonlinear Phenomena, vol. 65, No. 1-2, pp. 117-134, 1993.

[8] A.R.M. Siddique et al. "A comprehensive review on vibration based micro power generators using electromagnetic and piezoelectric transducer mechanisms", Energy Conversion and Management, 106, pp.728-747, 2015. 